\title{
BRANCHED STRUCTURES ON RIEMANN SURFACES
}

\author{
BY \\ RICHARD MANDELBAUM( $\left.{ }^{1}\right)$
}

\begin{abstract}
Following results of Gunning on geometric realizations of projective structures on Riemann surfaces, we investigate more fully certain generalizations of such structures.

We define the notion of a branched analytic cover on a Riemann surface $M$ (of genus $g$ ) and specialize this to the case of branched projective and affine structures. Establishing a correspondence between branched projective and affine structures on $M$ and the classical projective and affine connections on $M$ we show that if a certain linear homogeneous differential equation involving the connection has only meromorphic solutions on $M$ then the connection corresponds to a branched structure on $M$. Utilizing this fact we then determine classes of positive divisors on $M$ such that for each divisor $\mathfrak{D}$ in the appropriate class the branched structures having $\mathfrak{D}$ as their branch locus divisor form a nonempty affine variety. Finally we apply some of these results to study the structures on a fixed Riemann surface of genus 2 .
\end{abstract}

1. Introduction. The classical [1], [3], [5], [6], [14] representation of a Riemann surface as the quotient space of a subspace of the complex projective line $\boldsymbol{P}$ by a Fuchsian group provides a special coordinate covering of the Riemann surface, with the property that the local analytic coordinates are related to one another by projective transformations. Conversely, [1], [3], [7], [8] in general, a special coordinate covering can be realized geometrically as a fixed point free Fuchsian group acting on a subspace of $\boldsymbol{P}$. The question arises as to what type of coordinate cover would admit a realization corresponding to Fuchsian groups with elliptic elements. The natural answer would seem to be coordinate covers which admit branched coordinate functions. The aim of this paper is to investigate the nature of such branched covers. In addition we also investigate how branching affects the existence of affine covers, which in the unbranched case exist only for open Riemann surfaces and compact surfaces of genus one.

Briefly the contents of the paper are the following. $\S 2$ develops the notation and terminology to be used and defines the structures to be studied. $\S 3$ establishes a correspondence between branched projective and affine structures on the Riemann surface $M$ (of genus $g$ ) and the classical projective and affine connections on $M$.

Presented to the Society, April 9, 1971; received by the editors January 14, 1971 and, in revised form, May 19, 1971.

AMS 1970 subject classifications. Primary 30A46, 30A52; Secondary 32G15, 30A58.

Key words and phrases. Branched structures, Riemann surfaces, affine structure, projective structure, affine connection, projective connection, divisor, affine variety, quadratic differentials, abelian differentials, Weierstrass points.

(1) A major portion of this paper was first produced as part of my thesis at Princeton University under the guidance of R. C. Gunning. I wish to reiterate my appreciation for his help.

Copyright (C) 1972, American Mathematical Society 
In $\S \S 4$ and 5 we establish the existence of linear homogeneous differential equations on $M$ involving the projective and affine connections, respectively, whose solution spaces determine whether a specified branched structure on $M$ exists or not. Utilizing this fact we then determine classes of positive divisors on $M$ such that for each divisor $\mathfrak{D}$ in the appropriate class, the branched projective or affine structures having $\mathfrak{D}$ as their branch locus divisor form an affine variety containing a submanifold of dimension $3 g-3$ in the projective case and $g$ in the affine case. Lastly, in $\S 6$ we apply some of these results to study the structures on a fixed Riemann surface of genus 2 .

2. Notation and terminology. A coordinate covering $\left\{U_{\alpha}, z_{\beta}\right\}$ of the two-dimensional manifold $M$ consists of an open covering $\left\{U_{\alpha}\right\}$ of $M$ together with homeomorphisms $z_{\alpha}: U_{\alpha} \rightarrow V_{\alpha}$ from the sets $U_{\alpha}$ to open subsets $V_{\alpha}$ of the complex line $C$. On each nonempty intersection $U_{\alpha} \cap U_{\beta}$ we can define coordinate transition functions

$$
f_{\alpha \beta}=z_{\alpha} \circ z_{\alpha \beta}^{-1}: z_{\beta}\left(U_{\alpha} \cap U_{\beta}\right) \rightarrow z_{\alpha}\left(U_{\alpha} \cap U_{\beta}\right),
$$

and we sometimes write our coverings $\left\{U_{\alpha}, z_{\alpha}, f_{\alpha \beta}\right\}$ if we wish to emphasize the transition functions.

As in $[1, \S 1]$ we can define $G$-covers on our manifold $M$, where $G$ is any pseudogroup of mappings of domains in $C$, by insisting that all the coordinate transition functions belong to $G$. We then define a $G$-structure on $M$ as an equivalence class of $G$-covers, where two $G$-covers $\left\{U_{\alpha}, z_{\alpha}\right\},\left\{W_{\alpha}, w_{\alpha}\right\}$ are equivalent if their union is a $G$-cover. If $G$ is the pseudo-group of differentiable or complex analytic maps we then simply have the standard differentiable 2-manifolds or complex 2-manifolds (i.e. Riemann surfaces).

We wish to generalize the concept of coordinate cover to include the possibility that $z_{\alpha}: U_{\alpha} \rightarrow V_{\alpha}$ is not exactly a homeomorphism but is instead a locally branched covering map. In order to achieve maximum generality we will also assume that the sets $V_{\alpha}$ lie in the projective line $\boldsymbol{P}$ rather than $\boldsymbol{C}$. Coordinate covers for which all the $V_{\alpha}$ are in $C$ will be called regular coordinate covers. Before continuing we recall the following facts about locally branched covering maps.

Let $M$ and $N$ be two-dimensional manifolds and let $f: M \rightarrow N$ be a continuous map. Then $f$ is a locally branched covering if and only if for each $p \in M$ there is an open neighborhood $U \subset M$ containing $p$, such that

$$
f \mid U-\{p\} \rightarrow f(U)-f(p)
$$

exhibits $U-p$ as an $m$-sheeted covering space for some integer $m$.

We let $O_{f}(p)=m-1$ and call this the branching order of the mapping $f$ at $p$. The point $p$ is regular if and only if $O_{f}(p)=0$, and a branch point of $f$ if $O_{f}(p)>0$. We note immediately that (i) the branch points are a discrete subset of $M$, finite if $M$ is compact, and (ii) $f$ is an open mapping and is a local homeomorphism in a neighborhood of any regular point. 
We call $\sum_{p \in M} O_{f}(p)$ the total branching order of $f$. If $f$ is constant we let $O_{f}(p)$ $=-\infty$.

Suppose $f: M \rightarrow N$ is any nontrivial complex analytic mapping between Riemann surfaces. Then it is known that $f$ is a locally branched covering and the branching order at a point $p$ is the order of the zero of the derivative of $f$ in any local regular coordinate system in $M$ and $N$, that is $O_{f}(p)=\nu_{p}\left(f^{\prime}\right)$.

Definition. Suppose $\left\{U_{\alpha}, z_{\alpha}\right\}$ is a coordinate covering of $M$. Suppose for each $\alpha$, $w_{\alpha}: U_{\alpha} \rightarrow Y_{\alpha}$ is a locally branched covering from the set $U_{\alpha}$ to the open subset $Y_{\alpha}$ of the projective line $\boldsymbol{P}$. Suppose further that for each nonempty intersection $U_{\alpha} \cap U_{\beta}$ there exists a homeomorphism

$$
\phi_{\alpha \beta}: w_{\beta}\left(U_{\alpha} \cap U_{\beta}\right) \rightarrow w_{\alpha}\left(U_{\alpha} \cap U_{\beta}\right)
$$

such that $w_{\alpha}(p)=\phi_{\alpha \beta} \circ w_{\beta}(p)$ for $p \in U_{\alpha} \cap U_{\beta}$. Then we say $\left\{U_{\alpha}, w_{\alpha}\right\}$ or $\left\{U_{\alpha}, w_{\alpha}, \phi_{\alpha \beta}\right\}$ is a branched cover on $M$.

We note that the existence of a branched cover on $M$ presupposes the existence of a coordinate cover on $M$. As in the case of coordinate covers we say two branched covers $\left\{U_{\alpha}, w_{\alpha}\right\},\left\{U_{\alpha}^{\prime}, w_{\alpha}^{\prime}\right\}$ are equivalent if their union is a branched cover and we define a branched structure as an equivalence class of branched covers. Also by restricting the homeomorphisms $\phi_{\alpha \beta}$ to suitable pseudo-groups $G$ we can also speak of branched $G$-structures on $M$. It is easily verifiable that if $\left\{U_{\alpha}, w_{\alpha}\right\},\left\{U_{\alpha}^{\prime}, w_{\alpha}^{\prime}\right\}$ are branched covers of $M$ then if $p \in U_{\alpha} \cap U_{\beta}$ then $O_{w_{\alpha}}(p)=O_{w_{\beta}}(p)$ and if $g_{\alpha}: w_{\alpha}\left(U_{\alpha}\right) \rightarrow w_{\alpha}^{\prime}\left(U_{\alpha}\right)$ is a homeomorphism such that $w_{\alpha}^{\prime}=g_{\alpha} \circ w_{\alpha}$ then $O_{w_{\alpha}}(p)$ $=O_{w_{\alpha}^{\prime}}(p)$. Therefore branch points are invariant under equivalence and we can speak of the branch points of a structure rather than of only a cover.

Now suppose $\left\{U_{\alpha}, w_{\alpha}, \phi_{\alpha \beta}\right\}$ is a branched cover of the Riemann surface $M$.

We say $\left\{U_{\alpha}, w_{\alpha}, \phi_{\alpha \beta}\right\}$ is an analytic branched cover of $M$ iff $w_{\alpha}$ is meromorphic on $U_{\alpha} \subset M$. This immediately implies that the $\phi_{\alpha \beta}$ are meromorphic. (If the $w_{\alpha}$ are holomorphic $\left\{U_{\alpha}, w_{\alpha}, \phi_{\alpha \beta}\right\}$ is a regular analytic branched cover.) If in addition all the $\phi_{\alpha \beta}$ are projective (resp. affine) transformations then we say $\left\{U_{\alpha}, w_{\alpha}, \phi_{\alpha \beta}\right\}$ is a projective (resp. affine) branched cover. In the obvious fashion we can also speak of analytic (resp. projective; resp. affine) branched structures on the Riemann surface $M$.

Lastly suppose $M$ is a Riemann surface with a branched analytic structure. Then we can always choose a representative branched cover $\left\{U_{\alpha}, w_{\alpha}\right\}$ such that

(i) each $U_{\alpha}$ has at most one branch point,

(ii) if $p$ is a branch point and $p \in U_{\alpha} \cap U_{\beta}$ then $\alpha=\beta$ and $w_{\alpha}(p)=0$,

(iii) if $U_{\alpha}$ has no branch points then $w_{\alpha}$ is a homeomorphism of $U_{\alpha}$ into a unit cell in $\boldsymbol{P}$.

We shall call such a cover a restricted cover on $M$ and will assume that all our covers are restricted.

We shall henceforth fix $M$ as a compact Riemann surface of genus $g \geqq 2$. 
3. Structures and connections. Having introduced the notion of affine and projective structures on a Riemann surface it remains to be seen under what circumstances a Riemann surface admits such structures and what is the nature of their branching. To investigate this question we introduce (Gunning [1], [3], [4]) the notion of an $\mathscr{F}_{\nu}$ connection $(\nu=1,2)$ on a Riemann surface.

We recall the definition of the differential operators $\theta_{i}: \mathscr{M}_{U} \rightarrow \mathscr{M}_{U}(i=1,2)$, $U \subset \boldsymbol{P}$; namely:

$$
\begin{aligned}
& \theta_{1} f(z)=f^{\prime \prime}(z) / f^{\prime}(z)=(d / d z)\left(\log f^{\prime}(z)\right) \quad \text { for } z \in U, \\
& \theta_{2} f(z)=\left(\theta_{1} f(z)\right)^{\prime}-\frac{1}{2}\left(\theta_{1} f(z)\right)^{2}=f^{\prime \prime}(z) / f^{\prime}(z)-\frac{3}{2}\left(f^{\prime \prime}(z) / f^{\prime}(z)\right)^{2}
\end{aligned}
$$

( $\theta_{2}$ is, of course, simply the Schwarzian derivative). We note that by computation (Gunning [2, Lemma 24]) we have that if $h=g \circ f$ and $w=f(z)$ then

$$
\theta_{v} h(z)=\theta_{v} g(w) \cdot f^{\prime}(z)^{v}+\theta_{v} f(z) \quad(\nu=1,2) .
$$

Letting $\mathscr{F}_{v}=\left\{f \mid\right.$ for some open subdomain $U \subset P ; f \in M_{U}$ and $\left.\theta_{v} f=0\right\}$ we immediately find that $\mathscr{F}_{1}$ is the complex affine group of dimension 1 , that is $\operatorname{GA}(1, C)$ while $\mathscr{F}_{2}=$ complex projective group of dimension $1=\operatorname{PL}(1, C)$.

Now suppose $\mathscr{U}=\left\{U_{\alpha}, z_{\alpha}, f_{\alpha \beta}\right\}$ is a representative unbranched complex analytic coordinate cover for the Riemann surface $M$. We can associate to $\mathscr{U}$ the cocycles $\left(\sigma_{\nu \alpha \beta}\right) \in Z^{1}\left(\mathscr{U}, \mathscr{M}\left(\kappa^{\nu}\right)\right),(\nu=1,2)$ (where $\kappa$ is the canonical line bundle on $M, \kappa_{\alpha \beta}(p)$ $\left.=\left(d z_{\beta} / d z_{\alpha}\right)_{p}\right)$ defined as follows.

$$
\begin{aligned}
& \sigma_{v \alpha \beta}\left(z_{\beta}(p)\right)=\theta_{v} f_{\alpha \beta}\left(z_{\beta}(p)\right) \quad \text { where } p \in U_{\alpha} \cap U_{\beta}, \\
& \sigma_{v \alpha \beta}\left(z_{\gamma}(p)\right)=\kappa_{\gamma \beta}^{v}(p) \cdot \sigma_{v \alpha \beta}\left(z_{\beta}(p)\right) \text { where } p \in U_{\alpha} \cap U_{\beta} \cap U_{\gamma} .
\end{aligned}
$$

By computation [1, p. 168] we see that $\left(\sigma_{v \alpha \beta}\right)$ is in fact a cocycle for $\nu=1,2$.

We then have

Definition. An $\mathscr{F}_{v}$ connection for the covering $\mathscr{U}$ is a zero cochain $h=\left(h_{\alpha}\right) \in$ $C^{0}\left(\mathscr{U}, \mathscr{M}\left(\kappa^{v}\right)\right)$ such that $\delta h=\sigma_{v}$.

Explicitly an $\mathscr{F}_{v}$ connection for $\mathscr{U}$ consists of sections $\left(h_{\alpha}\right) \in \Gamma\left(U_{\alpha}, \mathscr{M}\left(\kappa^{\nu}\right)\right)$ such that $\sigma_{v \alpha \beta}(p)=h_{\beta}(p)-h_{\alpha}(p)$ for $p \in U_{\alpha} \cap U_{\beta}$, or in terms of the local mapping function $z_{\alpha}$ on $U_{\alpha}$,

$$
\sigma_{\nu \alpha \beta}\left(z_{\beta}(p)\right)=h_{\beta}\left(z_{\beta}(p)\right)-\kappa_{\alpha \beta}^{-v} h_{\alpha}\left(z_{\alpha}(p)\right) .
$$

Using the usual notion of equivalence we call two connections $h, h^{\prime}$ for coverings $U, U^{\prime}$ equivalent if $h$ and $h^{\prime}$ can be extended to form a connection for $U \cup U^{\prime}$. An equivalence class of connections will be called an $\mathscr{F}_{v}$ connection for $M$. An $\mathscr{F}_{1}$ connecting being simply the classical affine connection while an $\mathscr{F}_{2}$ connection is just a projective connection [4].

Definition. Suppose $\tau=\left\{\tau_{\alpha}\right\}$ is an $\mathscr{F}_{v}$ connection for $\mathscr{U}$. Suppose further that there exist meromorphic sections $\left\{h_{\alpha}\right\} \in C^{0}(\mathscr{U}, \mathscr{M})$ such that if $\tau$ and $h$ are realized as functions $h_{\alpha}\left(z_{\alpha}\right), \tau_{\alpha}\left(z_{\alpha}\right)$ on $z_{\alpha}\left(U_{\alpha}\right)$, then

$$
\theta_{v} h_{\alpha}\left(z_{\alpha}(p)\right)=\tau_{\alpha}\left(z_{\alpha}(p)\right) \text {. }
$$

We will then call $\tau$ an integrable $\mathscr{F}_{\nu}$ connection. 
THEOREM 1. There is a canonical bijection between the integrable $\mathscr{F}_{v}$-connections and the $\mathscr{F}_{v}$-structures on $M$.

Proof. We first need the following lemma.

Lemma 1. Suppose $h, k$ are $\mathscr{F}_{v}$-connections for $M$. Then $h=k+\tau$ for some $\tau=\left(\tau_{\alpha}\right) \in \Gamma\left(M, \mathscr{M}\left(\kappa^{v}\right)\right)$.

Proof of lemma. Representing $h$ by $\left\{h_{\alpha}\right\}$ and $k$ by $\left\{k_{\alpha}\right\}$, we let $\tau_{\alpha}=h_{\alpha}-k_{\alpha}$. We then note that since $\left\{\tau_{\alpha}\right\} \in C^{0}\left(\mathscr{U}, \mathscr{M}\left(\kappa^{v}\right)\right)$ and by computation $\delta\left(\tau_{\alpha}\right)=0 ;\{\tau\}$ is in fact in $\Gamma\left(M, \mathscr{M}\left(\kappa^{\nu}\right)\right)$. (In words: The difference between two projective (resp. affine) connections is a meromorphic quadratic (resp. abelian) differential.)

Proof of theorem. Let $\left\{U_{\alpha}, z_{\alpha}, f_{\alpha \beta}\right\}$ be a representative complex analytic coordinate cover for $M$.

Suppose $\left\{U_{\alpha}, w_{\alpha}, T_{\alpha \beta}\right\}$ represents an $\mathscr{F}_{v}$ structure on $M$. Thus in $U_{\alpha}, w_{\alpha}=h_{\alpha}\left(z_{\alpha}\right)$ for some $h_{\alpha} \in \mathscr{M}_{z_{\alpha}\left(U_{\alpha}\right)}$. Now let $\tau_{\alpha}\left(z_{\alpha}(p)\right)=\theta_{v} h_{\alpha}\left(z_{\alpha}(p)\right)$ for $p \in U_{\alpha}$. We claim $\tau=\left\{\tau_{\alpha}\right\}$ will be an integrable $\mathscr{F}_{v}$-connection. We note that $h_{\alpha} \circ f_{\alpha \beta}\left(z_{\beta}(p)\right)=T_{\alpha \beta} \circ h_{\beta}\left(z_{\beta}(p)\right)$ for $p \in U_{\beta}$. Thus by (3)

$$
\theta_{v} h_{\alpha}\left(z_{\alpha}\right) \cdot f_{\alpha \beta}^{\prime v}\left(z_{\beta}\right)+\theta_{v} f_{\alpha \beta}\left(z_{\beta}\right)=\theta_{\nu} T_{\alpha \beta}\left(w_{\beta}\right) \cdot h_{\beta}^{\prime v}\left(z_{\beta}\right)+\theta_{v} h_{\beta}\left(z_{\beta}\right) .
$$

However $\left\{U_{\alpha}, w_{\alpha}, T_{\alpha \beta}\right\}$ is an $\mathscr{F}_{v}$-structure, so that $\theta_{v} T_{\alpha \beta}\left(w_{\beta}\right)=0$. So we have

$$
\tau_{\alpha}\left(z_{\alpha}\right) \cdot \kappa_{\alpha \beta}^{-v}(p)+\sigma_{v \alpha \beta}\left(z_{\beta}\right)=\tau_{\beta}\left(z_{\beta}\right),
$$

where $\kappa_{\alpha \beta}(p)=\left[f_{\alpha \beta}^{\prime}\left(z_{\beta}(p)\right)\right]^{-1}$, and therefore $\left\{\tau_{\alpha}\right\}$ is an $\mathscr{F}_{v}$ connection which is obviously integrable. Furthermore any equivalent $\mathscr{F}_{v}$-cover $\left\{U_{\alpha}, \tilde{w}_{\alpha}, \tilde{T}_{\alpha \beta}\right\}$ will give rise to an equivalent connection, since if $\left\{U_{\alpha}, \tilde{w}_{\alpha}, \tilde{T}_{\alpha \beta}\right\}$ is equivalent to $\left\{U_{\alpha}, w_{\alpha}, T_{\alpha \beta}\right\}$, then $\tilde{w}_{\alpha}=T_{\alpha} \circ w_{\alpha}$ for some $T_{\alpha} \in \mathscr{F}_{v}$, and therefore $\theta_{v} \tilde{w}_{\alpha}=\theta_{v} w_{\alpha}$.

Now suppose $\tau \in C^{0}\left(\mathscr{U}, \mathscr{M}\left(\kappa^{\nu}\right)\right)$ is an integrable $\mathscr{F}_{\nu}$-connection; and let $\left\{h_{\alpha}\right\} \in$ $C^{0}(U, M)$ be such that $\theta_{v} h_{\alpha}\left(z_{\alpha}\right)=\tau_{\alpha}\left(z_{\alpha}\right)$. Let $w_{\alpha}=h_{\alpha}\left(z_{\alpha}\right)$. We claim that $\left\{U_{\alpha}, w_{\alpha}\right\}$ will define an $\mathscr{F}_{v}$-structure which will in turn correspond to the connection $\tau$ under the construction in the first part of the proof.

We can assume $\left\{U_{\alpha}\right\}$ is a restricted cover and therefore define transition functions $T_{\alpha \beta}$ for $\left\{U_{\alpha}, w_{\alpha}\right\}$ by means of inverses $T_{\alpha \beta}=w_{\alpha} \circ w_{\beta}^{-1}$ in $U_{\alpha} \cap U_{\beta}$.

$$
T_{\alpha \beta} \circ h_{\beta}=\left(h_{\alpha} \circ z_{\alpha}\right) \circ\left(h_{\beta} \circ z_{\beta}\right)^{-1} \circ h_{\beta}=h_{\alpha} \circ f_{\alpha \beta} \circ h_{\beta}^{-1} \circ h_{\beta}=h_{\alpha} \circ f_{\alpha \beta} .
$$

Then applying (3) we have

$$
\theta_{\nu} T_{\alpha \beta}\left(w_{\beta}\right) \cdot h_{\beta}^{\prime}\left(z_{\beta}\right)^{v}=\left[\tau_{\beta}\left(z_{\beta}\right)-\kappa_{\alpha \beta}^{-v}(p) \tau_{\alpha}\left(z_{\alpha}\right)\right]-\sigma_{v \alpha \beta}\left(z_{\beta}\right) .
$$

But $\tau$ is an $\mathscr{F}_{v}$ connection, so

$$
\left[\theta_{v} T_{\alpha \beta}\left(w_{\beta}\right)\right]\left[h_{\beta}^{\prime}\left(z_{\beta}\right)\right]^{v} \equiv 0 \quad \text { in } U_{\beta} .
$$

However $h_{\beta}^{\prime}\left(z_{\beta}\right)=0$ at most at one point in $U_{\beta}$ and $\theta_{v} T_{\alpha \beta}$ is meromorphic so $\theta_{v} T_{\alpha \beta} \equiv 0$ in $U_{\beta}$, and therefore $\left\{U_{\alpha}, z_{\alpha}, T_{\alpha \beta}\right\}$ is an $\mathscr{F}_{v}$-cover. So each $\mathscr{F}_{\nu}$ cover corresponds uniquely to an $\mathscr{F}_{v}$-connection and we can immediately verify that this bijective correspondence preserves equivalence, thus proving the theorem. 
4. Branched projective structures. Suppose $\left\{U_{\alpha}, w_{\alpha}\right\}$ is a branched analytic cover on $M$. We can then canonically associate to it the positive divisor $\mathscr{D}_{\left\{U_{\alpha}, w_{\alpha}\right\}}=$ $\sum_{p \in M} O_{w_{\alpha}}(p) \cdot p$ for some $\alpha$ such that $p \in U_{\alpha}$. Since $M$ is compact $\sum_{p \in M} O_{w_{\alpha}}(p) \cdot p$ is finite and therefore recalling that equivalence of covers preserves branch points we have a map $\mathfrak{D}:\{$ structures on $M\} \rightarrow\{$ positive divisors on $M$ \}. Given a positive divisor $\mathfrak{D}$ we shall then say the branched analytic cover $\left\{U_{\alpha}, w_{\alpha}\right\}$ is of type $\mathfrak{D}$ if and only if $\mathfrak{D}_{\left\{U_{\alpha}, w_{\alpha}\right\}}=\mathfrak{D}$. We let $B_{\left\{U_{\alpha}, w_{\alpha}\right\}}=B\left(\left\{U_{\alpha}, w_{\alpha}\right\}\right)=B\left(\mathfrak{D}_{\left\{U_{\alpha}, w_{\alpha}\right\}}\right)=B(\mathfrak{D})=\sum_{p \in M} O_{w_{\alpha}}(p)$ and call this the total order or total branching order of the structure. We shall also let $\left|\mathscr{D}_{\left\{U_{\alpha}, w_{\alpha}\right\}}\right|=\left|\left\{U_{\alpha}, w_{\alpha}\right\}\right|=$ card $\left\{p_{j} \in M \mid O_{w_{\alpha}}\left(p_{j}\right) \neq 0\right\}$. This will be called the multiplicity of the structure.

Lastly if $\mathfrak{D}$ is a positive divisor such that $\mathfrak{D}=\sum_{j=1}^{j=n}\left(r_{j}-1\right) \cdot p_{j}, r_{j}>1$; we shall say that a projective connection $\tau$ on $M$ is of type $\mathfrak{D}$ if and only if for each $p_{j}$ we have (assuming $\left.z_{\alpha}\left(p_{j}\right)=0\right)$

$$
\tau_{\alpha}\left(z_{\alpha}\right)=\frac{1-r_{j}^{2}}{2 z_{\alpha}^{2}}+t_{-1} z_{\alpha}^{-1}+\sum_{i=0}^{\infty} t_{i} z_{\alpha}^{i}
$$

with $\sum t_{i} z_{\alpha}^{i}$ convergent, in a neighborhood $U_{\alpha}$ of $p_{j}$, while $\tau_{\alpha}$ is holomorphic in a neighborhood of any nonbranch point.

This last definition is justified by

Proposition 2. For each positive divisor $\mathfrak{D}$ on $M$ there exists a canonical bijection between the projective structures of type $\mathfrak{D}$ on $M$ and the integrable projective connections of type $\mathfrak{D}$ on $M$.

Proof. It suffices to show that if $f: U \rightarrow V$ is a meromorphic function with $O \in U$ and $O_{f}(0)=r-1, r$ an integer $\geqq 1$, then $\theta_{2} f(z)=\left(1-r^{2}\right) / 2 z^{2}+t_{-1} / z+A(z)$ where $t_{-1}=0$ if $r=1$ and $A$ is analytic in $U$. However if $O_{f}(0)=r-1$ then we can write locally $f(z)=(g(z))^{ \pm r}$ where $g(0)=0 ; g^{\prime}(0) \neq 0$. But then by direct calculation we see that $\theta_{2} f(z)=\left(\left(1-r^{2}\right) / 2\right)\left(g^{\prime}(z) / g(z)\right)^{2}+\theta_{2} g(z)$. Thus $g^{\prime}(z) / g(z)=1 / z+$ (holomorphic function) if $r>1$ while $\theta_{2} f(z)=\theta_{2} g(z)=$ (holomorphic function) if $r=1$, proving the theorem.

We will call a projective connection $\tau$ holomorphic if it has a representative $\left\{\tau_{\alpha}\right\}$ where all the $\tau_{\alpha}$ are holomorphic. We then have as an obvious consequence of the proposition,

COROLlaRY 2.1 (SEE [1], [3]). There is a canonical bijection between the unbranched projective structures on $M$ and the integrable holomorphic projective connections on $M$.

We thus have shown that the study of projective structures of a given type is equivalent to the study of integrable projective connections of that type. Let us then fix $\mathfrak{D}$ as a positive divisor with the expansion $\mathfrak{D}=\sum_{j=1}^{j=n}\left(r_{j}-1\right) \cdot p_{j} ; r_{j}>1$ on $M$. We then fix $n$ as the multiplicity of $\mathscr{D}$ and $B$ as the total branching order. 
THEOREM 3. (i) The integrable projective connections of type $\mathfrak{D}$ have the structure of a complex affine subvariety of the $3 g-3+n$-dimensional complex linear manifold of all projective connections of type $\mathfrak{D}$.

(ii) Furthermore if $n=1$ or $B \leqq 2 g-2$ then this subvariety is nonempty and its regular points form a $3 g$-3-dimensional submanifold of the space of all projective connections of type $\mathfrak{D}$.

Proof. We will first show that the space of all projective connections of type $\mathfrak{D}$ forms a $3 g-3+n$-dimensional complex linear manifold. We recall that by Lemma 1 , given a projective connection, we can produce all other projective connections by simply adding appropriate quadratic differentials. Thus suppose $\tau$ is a projective connection of type $\mathfrak{D}$. By adding to $\tau$ any quadratic differential which is holomorphic except for at most simple poles at $p_{j}$ we produce all the projective connections of type $\mathfrak{D}$. That is

\{projective connections of type $\mathfrak{D}\}$

$=\tau+\left\{\right.$ quadratic differentials with at most simple poles at $\left.p_{j},(j=1, \ldots, n)\right\}$.

However by the Riemann-Roch theorem the space of quadratic differentials with at most simple poles at $p_{j},(j=1, \ldots, n)$ is isomorphic to a complex number space of dimension $3 g-3+n$. Thus if there exists one projective connection of type $\mathfrak{D}$, then there exists a $3 g-3+n$-complex linear manifold of such connections. However by [3, Corollary 2 to Theorem 4] we see that there exists a projective connection $\sigma$ of type 0 on $M$. Now utilizing the notation of [1], [2] and [3] $\Gamma\left(M, \mathcal{O}_{m}\left(\kappa^{2},-2 \cdot p_{j}\right)\right)$ is the space of quadratic differentials with at most a double pole at $p_{j}$ while $\Gamma\left(M, \mathcal{O}_{m}\left(\kappa^{2},-1 \cdot p_{j}\right)\right)$ is the space of quadratic differentials with at most a single pole at $p_{j}$. However by the Riemann-Roch theorem

$$
\operatorname{dim} \Gamma\left(M, \mathcal{O}_{m}\left(\kappa^{2},-2 \cdot p_{j}\right)\right)=3 g-1 \text { and } \operatorname{dim} \Gamma\left(M, \mathcal{O}_{m}\left(\kappa^{2},-1 \cdot p_{j}\right)\right)=3 g-2 .
$$

Therefore for each $p_{j}$ there exists a quadratic differential $\theta_{j}$ which has exactly a pole of order 2 at $p_{j}$ and no other singularities. Letting $a_{j}$ be the coefficient of $z_{\alpha_{j}}^{-2}$ in the Laurent expansion of $\theta_{j}$ around $z_{\alpha}\left(p_{j}\right)=0$ we let $\theta=\sum_{j=1}^{j=n}\left(\left(1-r_{j}^{2}\right) / 2 a_{j}\right) \theta_{j}$. Then it is immediately apparent that $\theta$ is a quadratic differential such that $\tau=\sigma+\theta$ is a projective connection of type $\mathscr{D}$. We are therefore left with the task of showing that integrable projective connections of type $\mathfrak{D}$ form a complex affine subvariety.

Let $F_{2}(M, \mathfrak{D})$ be the space of projective connections of type $\mathfrak{D}$. Let $\tau \in F_{2}(M, \mathfrak{D})$. Let us now choose a basis for the vector space of quadratic differentials with at most a simple pole at $p_{j}, j=1, \ldots, n$. By the constructions we made previously we can choose this basis as follows:

Let ${ }_{j} q, j=1, \ldots, 3 g-3$, be a basis for the space of holomorphic quadratic differentials. Let ${ }_{j} \theta$ be a quadratic differential which is holomorphic everywhere except at $p_{j}$ where it has the expansion ${ }_{j} \theta\left(z_{\alpha}\right)=\left(1 / z_{\alpha}\right)+\sum_{i=0}^{\infty} \theta(\alpha)_{i} z_{\alpha}^{i}$. We let $\boldsymbol{q}=$ $\left({ }_{1} q, \ldots,{ }_{3 g-3} q\right), \boldsymbol{\theta}=\left({ }_{1} \theta, \ldots,{ }_{n} \theta\right)$, and for each $(\boldsymbol{t}, \boldsymbol{\sigma})=\left(t_{1}, \ldots, t_{n}, \sigma_{1}, \ldots, \sigma_{3 g-3}\right)$ 
$\in C^{n} \times C^{3 g-3}$, we let ${ }_{(t, \sigma)} \mu=\tau+\theta \cdot t+q \cdot \sigma$ be the element of $F_{2}(M, \mathfrak{D})$ determined by $(t, \sigma)$. Therefore we see that

$$
I F_{2}(M, \mathfrak{D})=\{\text { integrable projective connections of type } \mathfrak{D}\}
$$

is equivalent to

$\left\{(\boldsymbol{t}, \boldsymbol{\sigma}) \in \boldsymbol{C}^{n} \times \boldsymbol{C}^{3 g-3} \mid \theta_{2} w_{\alpha}\left(z_{\alpha}\right)={ }_{(\boldsymbol{t}, \sigma)} \mu_{\alpha}\left(z_{\alpha}\right)\right.$ has a meromorphic solution for all $\left.\alpha\right\}$.

Following [1], [13] we note that the problem of solving

$$
\theta_{2} w(z)=S(z)
$$

for meromorphic $S(z)$ is equivalent to the simpler problem of finding two linear independent solutions $w_{1}$ and $w_{2}$ of the linear homogeneous second order equation

$$
w^{\prime \prime}(z)+\frac{1}{2} S(z) w(z)=0 .
$$

That is, if $w_{1}$ and $w_{2}$ solve (5) then $w_{1} / w_{2}$ solves (4), the general solution being $\left(a w_{1}+b w_{2}\right) /\left(c w_{1}+d w_{2}\right)$ with $a d-b c=1$. Furthermore it is immediately verifiable that if $S(z)$ is holomorphic then so are $w_{1}$ and $w_{2}$ and a solution $w_{h}$ to (4) can be found such that $w_{h}$ is holomorphic and $w_{h}^{\prime}(z) \neq 0$. Thus to determine $I F_{2}(M, \mathfrak{D})$ we must find out what

$\left\{(\boldsymbol{t}, \boldsymbol{\sigma}) \in \boldsymbol{C}^{n} \times \boldsymbol{C}^{3 g-3} \mid w_{\alpha}^{\prime \prime}\left(z_{\alpha}\right)+\frac{1}{2}(\boldsymbol{t}, \sigma) \mu_{\alpha}\left(z_{\alpha}\right)=0\right.$ has two linearly independent meromorphic solutions in each $U_{\alpha}$ containing a $p_{j}$ \}

is. Now we recall that in a neighborhood $U_{\alpha}$ of $p_{j}$,

$$
{ }_{(t, \sigma)} \mu_{\alpha}\left(z_{\alpha}\right)=\frac{1-r_{j}^{2}}{2 z_{\alpha}^{2}}+(t, \sigma) \mu_{-1}(\alpha) z_{\alpha}^{-1}+\sum_{i=0}^{\infty}(t, \sigma) \mu_{i}(\alpha) z_{\alpha}^{i}
$$

Thus by [12], [13] there exists a polynomial $A\left(r_{j}\right)$ in $C\left[x_{1}, \ldots, x_{r}\right]$ (where

$$
A(r)\left(x_{1}, \ldots, x_{r}\right)=\sum_{\left(i_{1}, \ldots, i_{r}\right)} \alpha_{\left(i_{1}, \ldots, i_{r}\right)} x_{1}^{i_{1}} \cdots x_{r}^{i_{r}}
$$

and $\left(i_{1}, \ldots, i_{r}\right)$ are such that $\left.\sum_{k=1}^{k=r} k i_{k}=r\right)$; with leading term $\alpha_{1} x_{1}^{r}$ (with $\alpha_{1} \neq 0$ ) such that $w_{\alpha}^{\prime \prime}\left(z_{\alpha}\right)+\frac{1}{2}(t, \sigma) \mu_{\alpha}\left(z_{\alpha}\right)=0$ has two linearly independent meromorphic solutions in $U_{\alpha}$ if and only if

$$
A\left(r_{j}\right)\left(_{(t, \sigma)} \mu_{-1}(\alpha), \ldots,{ }_{(t, \sigma)} \mu_{r-2}(\alpha)\right)=0 .
$$

However $_{(t, \sigma)} \mu_{-1}(\alpha)=\tau_{-1}(\alpha)+t_{1}$ and, for $k \geqq 0,{ }_{(t, \sigma)} \mu_{k}(\alpha)=\tau_{k}(\alpha)+\theta_{k}(\alpha) \cdot t+q_{k}(\alpha) \cdot \sigma$ where $\tau_{k}(\alpha), \theta_{k}(\alpha), q_{k}(\alpha)$ are just the coefficients of $z_{\alpha}^{k}$ in the Laurent expansion around $z_{\alpha}\left(p_{j}\right)=0$ of $\tau(\alpha), \theta(\alpha), q(\alpha)$ respectively.

Thus there exists a polynomial $K_{j} \in C\left[x_{1}, \ldots, x_{3 g-3+n}\right]$ with leading term $\alpha_{0}^{(j)} x_{j}^{r_{j}}$, $\alpha_{0}^{(j)} \neq 0$; and total degree of any other term $\leqq\left[r_{j} / 2\right]$; such that

$$
\left.A\left(r_{j}\right){ }_{(t, \sigma)} \mu_{-1}(\alpha), \ldots,{ }_{(t, \sigma)} \mu_{r-2}(\alpha)\right)=0 \quad \text { iff } K_{j}(t, \sigma)=0 .
$$


Then the $K_{j}(j=1, \ldots, n)$ form a system of $n$-polynomials in $n+3 g-3$ variables. If we now let

$$
{ }_{p} V_{\mathfrak{D}}=\left\{(\boldsymbol{t}, \boldsymbol{\sigma}) \in C^{n} \times C^{3 g-3} \mid K_{j}(\boldsymbol{t}, \boldsymbol{\sigma})=0, j=1, \ldots, n\right\},
$$

then $I F_{2}(M, \mathfrak{D}) \approx{ }_{p} V_{\mathfrak{D}}$ which is the required subvariety.

Furthermore if we look at the mapping $K: C^{3 g-3+n} \rightarrow C^{n}$ given by $K(t, \sigma)$ $=\left(K_{1}(t, \sigma), \ldots, K_{n}(t, \sigma)\right)$ we can show by a direct calculation that "in general" the rank of $J(K)$, the Jacobian matrix of the mapping $K$, will be $n$. Therefore if ${ }_{p} V_{\mathfrak{D}}^{0} \subset{ }_{p} V_{\mathfrak{D}}$ consists of the regular points of ${ }_{p} V_{\mathfrak{D}}$ then if ${ }_{p} V_{\mathfrak{D}} \neq 0,{ }_{p} V_{\mathfrak{D}}^{0}$ is in turn a submanifold of dimension $3 g-3$. We see immediately that if $n=0,{ }_{p} V_{\mathfrak{D}}=C^{3 g-3}$, and if $n=1,{ }_{p} V_{\mathfrak{D}}$ is just a union of hypersurfaces in $C^{3 g-2}$.

Now suppose $B \leqq 2 g-2$. We will show ${ }_{p} V_{\mathfrak{D}} \neq 0$, thus concluding our theorem.

Suppose for each $j$, we could pick a quadratic differential ${ }_{j} \theta$ such that ${ }_{j} \theta$ is holomorphic everywhere except that

(i) around $p_{j},{ }_{j} \theta\left(z_{\alpha}\right)=1 / z_{\alpha}+\sum_{i=0}^{\infty}{ }_{j} \theta(\alpha)_{i} z_{\alpha}^{i}$ and

(ii) around $p_{i} \neq p_{j},{ }_{j} \theta\left(z_{\beta}\right)=z_{\beta}^{r_{i}-1} \sum_{k=0}^{\infty}, \theta(\beta)_{k} z_{\beta}^{k}$.

The system ${ }_{j} q, j=1, \ldots, 3 g-3,{ }_{j} \theta, j=1, \ldots, n$, will again form a basis for the quadratic differentials with at most a simple point at $p_{j}$. However if we now calculate $K_{j}(t, \sigma)$ we find that, since the ${ }_{i} \theta$ for $i \neq j$ have no terms of degree less than $r_{j}-1$ around $p_{j}, K_{j}(\boldsymbol{t}, \boldsymbol{\sigma})=K_{j}\left(\left(0, \ldots, t_{j}, \ldots, 0\right), \boldsymbol{\sigma}\right)$. Thus for each $j, K_{j}$ will be a polynomial in $t_{j}$ and $\sigma$ with leading term $\alpha_{0}^{(j)} t_{j}^{r j}, a_{0}^{(j)} \neq 0$. Thus for any fixed $\sigma$, if we let $t_{j}^{*}$ be a root of $K_{j}\left(t_{j}, \sigma\right)=0$ for $j=1, \ldots, n$ we immediately see that $\left(t^{*}, \sigma\right) \in{ }_{p} V$ where $t^{*}=\left(t_{1}^{*}, \ldots, t_{n}^{*}\right)$. Therefore ${ }_{p} V_{\mathfrak{D}} \neq 0$.

So we must prove that we can pick the ${ }_{j} \theta$ as described above.

Now let $\phi_{j}$ be the divisor $\left(r_{1}-1\right) \cdot p_{\imath}+\cdots+(-1) \cdot p_{j}+\cdots+\left(r_{n}-1\right) \cdot p_{n}$. Let $Q(\mathfrak{D})$, for any divisor $\mathscr{D}$, be the space of quadratic differentials whose divisors are multiples of $\mathfrak{D}$.

Then if for each $j, Q\left(\phi_{j}\right)-Q\left(\phi_{j}+p_{j}\right) \neq 0$, we have our basis, since if we let ${ }_{j} \psi \in Q\left(\phi_{j}\right)-Q\left(\phi_{j}+p_{j}\right)$ for each $j$, then we simply let ${ }_{j} \theta={ }_{j} \psi / \operatorname{Res}_{p_{j}}\left({ }_{j} \psi\right)$ and the ${ }_{j} \theta$, $j=1, \ldots, n$, are as required.

However by the Riemann-Roch theorem provided deg $\left(\phi_{j}\right) \leqq 2 g-4$ we have

$$
\operatorname{dim} Q\left(\phi_{j}\right)=3 g-3-\operatorname{deg}\left(\phi_{j}\right) \geqq g+1
$$

while

$$
\operatorname{dim} Q\left(\phi_{j}+p_{j}\right)=3 g-3-\operatorname{deg}\left(\phi_{j}+p_{j}\right)=3 g-3-\operatorname{deg}\left(\phi_{j}\right)-1 \geqq g .
$$

Thus $\operatorname{dim} Q\left(\phi_{j}\right)-\operatorname{dim} Q\left(\phi_{j}+p_{j}\right) \geqq 1$ and $Q\left(\phi_{j}\right)-Q\left(\phi_{j}+p_{j}\right) \neq 0$, provided $\operatorname{deg}\left(\phi_{j}\right)$ $\leqq 2 g-4$. However

$$
\operatorname{deg}\left(\phi_{j}\right)<\operatorname{deg}\left(\phi_{j}+p_{j}\right)<\operatorname{deg}\left(\phi_{j}+p_{j}^{r_{1}}\right)=B \leqq 2 g-2 .
$$

Thus deg $\left(\phi_{j}\right) \leqq 2 g-4$ and our theorem is proven.

We note that our result can be extended to $B \leqq 3 g-2$ provided the points $p_{1} \cdots p_{n}$ avoid a one-codimensional subset $A \subset M^{n}$ (see [1], [10] and [11]). 
5. Branched affine structures. Having discussed $\mathscr{F}_{v}$ structures and connections for $\nu=2$ we now turn to the case of $\nu=1$, the affine structures. So suppose $h=$ $\left(h_{\alpha}\right) \in C^{0}(\mathscr{U}, \mathscr{M}(\kappa))$ is an affine connection for $U$. We recall that this means

$$
\sigma_{1 \alpha \beta}\left(z_{\beta}\right)=f_{\alpha \beta}^{\prime \prime}\left(z_{\beta}\right) / f_{\alpha \beta}^{\prime}\left(z_{\beta}\right)=\left(d / d z_{\beta}\right) \log f_{\alpha \beta}^{\prime}=h_{\beta}\left(z_{\beta}\right)-\kappa_{\alpha \beta}^{-1} h_{\alpha}\left(z_{\alpha}\right)
$$

where $\mathscr{U}=\left\{U_{\alpha}, z_{\alpha}, f_{\alpha \beta}\right\}$ is a coordinate cover of $M$.

Paralleling the development in [9] we now let $p \in U_{\alpha} \cap U_{\beta}$ and let $\Gamma \subset U_{\alpha} \cap U_{\beta}$ be a simple closed curve bounding a cell $D \ni p$ in $U_{\alpha} \cap U_{\beta}$. We then define

$$
\operatorname{Res}(h, p)=\frac{1}{2 \pi i} \int_{\Gamma} h_{\alpha} d z_{\alpha}
$$

as the residue of $h$ at $p$, and

$$
\operatorname{Res}(h)=\sum_{p \in M} \operatorname{Res}(h, p) .
$$

We immediately note that $\operatorname{Res}(h, p)$ is independent of the choice of representatives for $h$ since

$$
d \log f_{\alpha \beta}^{\prime}\left(z_{\beta}\right)+h_{\alpha}\left(z_{\alpha}\right) d z_{\alpha}=h_{\beta}\left(z_{\beta}\right) d z_{\beta},
$$

and $f_{\alpha \beta}^{\prime} \neq 0$ in $U_{\alpha} \cap U_{\beta}$. Furthermore since any two affine connections $h, k$ differ at most by an abelian differential $\tau$, which has total residue 0 on the compact surface $M, \operatorname{Res}(h)=\operatorname{Res}(k)$. Thus the total residue of all affine connections are the same fixed constant. We then have

Lemma 2. Let $h=\left(h_{\alpha}\right)$ be an affine connection for $U$. Then $\operatorname{Res}(h)=2 g-2$.

Proof. Let $g=\left\{g_{\alpha}\right\} \in \Gamma(M, \mathscr{M}(\kappa))$ represent an abelian differential on $M$. Let

$$
k_{\alpha}\left(z_{\alpha}\right)=\left(d / d z_{\alpha}\right) \log g_{\alpha}\left(z_{\alpha}\right)=g_{\alpha}^{\prime}\left(z_{\alpha}\right) / g_{\alpha}\left(z_{\alpha}\right) .
$$

Now $g_{\alpha}\left(z_{\alpha}\right)=\kappa_{\alpha \beta}(p) g_{\beta}\left(z_{\beta}\right)$. Thus since $d / d z_{\beta}=\kappa_{\alpha \beta}^{-1}\left(d / d z_{\alpha}\right)$, we have

$$
\kappa_{\alpha \beta}^{-1}(p) \cdot k_{\alpha}\left(z_{\alpha}\right)=\left(d / d z_{\beta}\right) \log \kappa_{\alpha \beta}(p)+k_{\beta}\left(z_{\beta}\right) .
$$

But

$$
\sigma_{1 \alpha \beta}\left(z_{\beta}\right)=f_{\alpha \beta}^{\prime \prime}\left(z_{\beta}\right) / f_{\alpha \beta}^{\prime}\left(z_{\beta}\right)=-\left(d / d z_{\beta}\right) \log \kappa_{\alpha \beta}(p)
$$

Thus

$$
\sigma_{1 \alpha \beta}\left(z_{\beta}\right)=\kappa_{\beta}\left(z_{\beta}\right)-\kappa_{\alpha \beta}^{-1}(p) \cdot k_{\alpha}\left(z_{\alpha}\right)
$$

and therefore $k=\left\{k_{\alpha}\right\}$ is an affine connection for $U$. However

$$
\operatorname{Res}(k)=\frac{1}{2 \pi i} \sum_{p \in M} \int \frac{d}{d z_{\alpha}} \log g_{\alpha}\left(z_{\alpha}\right)=\sum_{p \in M} \nu_{p}\left(g_{\alpha}\right) .
$$

But since $g_{\alpha} \in \Gamma(M, \mathscr{M}(\kappa)), \sum_{p \in M} \nu_{p}\left(g_{\alpha}\right)=c(\kappa)=2 g-2$. So Res $(k)=2 g-2$ and, since all affine connections have the same residue, $\operatorname{Res}(h)=2 g-2$ also. 
Now suppose $\left\{U_{\alpha}, w_{\alpha}\right\}$ is a branched affine structure with a branch point of order $r-1$ at $p_{j}$. Thus locally in $U_{\alpha} \ni p_{j}$ we have

$$
w_{\alpha}=\left[g_{\alpha}\left(z_{\alpha}\right)\right]^{ \pm r} \text { where } g_{\alpha}: z_{\alpha}\left(U_{\alpha}\right) \rightarrow C
$$

with

$$
g_{\alpha}(0)=0, \quad g_{\alpha}^{\prime}(0) \neq 0, \quad \text { and } \quad z_{\alpha}\left(p_{j}\right)=0 .
$$

But affine transformations preserve poles so that, in contradistinction to the projective case, $\left[g_{\alpha}\left(z_{\alpha}\right)\right]^{+r}$ is not equivalent to $\left[g_{\alpha}\left(z_{\alpha}\right)\right]^{-r}$. Thus in addition to specifying the order of a branch point we must also specify whether it arises from a pole or not. To this end we introduce the notion of the "residue class" divisor $\chi$ as follows.

Suppose $\left\{U_{\alpha}, w_{\alpha}\right\}$ represents an affine structure on $M$ with poles of order $\nu_{j}$ at $S_{j}, j=1, \ldots, \sigma$, and nonpolar branch points of order $r_{j}-1$ at $p_{j}, j=1, \ldots, n$. (We note that $O_{w_{j}}\left(s_{j}\right)=\left|v_{j}\right|-1$.)

We now define the divisor (not necessarily positive)

$$
\chi_{\left(U_{\alpha}, w_{\alpha}\right\}}=\sum_{j=1}^{n}\left(r_{j}-1\right) \cdot p_{j}+\sum_{j=1}^{\sigma}\left(\nu_{j}-1\right) \cdot s_{j} .
$$

We thus have a map

$$
\chi:\{\text { affine structures on } M\} \rightarrow\{\text { divisors on } M\} \text {. }
$$

We let $\rho(\chi)=\sum_{j=1}^{j=n}\left(r_{j}-1\right)+\sum_{j=1}^{j=\sigma}\left(\nu_{j}-1\right)$ and $|\chi|=\eta(\chi)+\sigma(\chi)$ where if $\chi=\sum x_{j} \cdot p_{j}$ then

$$
\eta(\chi)=\operatorname{card}\left\{p_{j} \mid x_{j}>0\right\} \quad \text { and } \sigma(\chi)=\operatorname{card}\left\{p_{j} \mid x_{j}<0\right\} .
$$

Now given a divisor $\chi$ with $\rho(\chi)=2 g-2$ we shall say that an affine connection $h=\left(h_{\alpha}\right)$ is of class $\chi$ iff $h$ has only simple poles and

$$
\chi=\sum_{j=1}^{n} x_{j} \cdot p_{j} \Rightarrow \operatorname{Res}\left(h, p_{j}\right)=x_{j}
$$

If $\chi$ has no terms of the form $-1 \cdot p_{j}$ we shall say it is of the second kind.

We now have

Proposition 4. For each divisor of the second kind $\chi$ with $\rho(\chi)=2 g-2$ there is a canonical bijection between the affine structures of class $\chi$ on $M$ and the integrable affine connections of class $\chi$ on $M$.

Proof. It suffices to show that if $f: U \rightarrow V$ is a meromorphic function with $O \in U$ and $\nu_{0}\left(f^{\prime}\right)=r-1$ with $|r| \geqq 1$, then $\theta_{1} f(z)=(r-1) / z+A(z)$ where $A$ is analytic in a neighborhood of 0 . However if $\nu_{0}\left(f^{\prime}\right)=r-1$ we can write locally $f(z)=(g(z))^{ \pm r}$ with $g(0)=0, g^{\prime}(0) \neq 0$. But then by direct calculation

$$
\theta_{1} f(z)=(r-1)\left(g^{\prime}(z) / g(z)\right)+\theta_{1} g(z) .
$$


However $\theta_{1} g(z)$ is holomorphic and $g^{\prime}(z) / g(z)=1 / z+$ (holomorphic function). Thus $\theta_{1} f(z)=(r-1) / z+A(z)$ where $A(z)$ is holomorphic and $\theta_{1} f$ is holomorphic iff $r=1$.

We note that the proof implies that if $\left\{U_{\alpha}, w_{\alpha}\right\}$ represents an affine structure on $M$ and if $h$ is an affine connection corresponding to $\left\{U_{\alpha}, w_{\alpha}\right\}$ then necessarily $\chi_{\left\{U_{\alpha}, w_{\alpha}\right\}}$ is of the second kind with $\rho(\chi)=\operatorname{Res}(h)=2 g-2$.

We recall that a coordinate cover $\left\{U_{\alpha}, z_{\alpha}\right\}$ is regular iff $z_{\alpha}$ is holomorphic for each $\alpha$. Since holomorphicity is preserved by affine transformations we shall say $\left\{U_{\alpha}, w_{\alpha}\right\}$ represents a regular affine structure iff it is a regular affine cover. We immediately see that $\left\{U_{\alpha}, w_{\alpha}\right\}$ is regular iff $\chi_{\left\{U_{\alpha}, w_{\alpha}\right\}}$ is positive or equivalently iff $\chi_{\left\{U_{\alpha}, w_{\alpha}\right\}}=\mathscr{D}_{\left\{U_{\alpha}, w_{\alpha}\right\}}$.

Similarly we shall say that $h$ is a regular affine connection iff $\operatorname{Res}(h, p) \geqq 0$ for all $p \in M$.

THEOREM 5. Let $\mathfrak{D}$ be a positive divisor with $B(\mathfrak{D})=2 g-2$. Then the integrable affine connections of class $\mathfrak{D}$ form a g-dimensional complex linear manifold.

Proof. Suppose $h$ is an affine connection of class $\mathfrak{D}$. Then so is $h+\tau$ for any holomorphic abelian differential $\tau$. But the space of holomorphic abelian differentials is isomorphic to $\boldsymbol{C}^{g}$. Thus it suffices to show that

(i) there exists an affine connection of class $\mathfrak{D}$,

(ii) any affine connection of class $\mathfrak{D}$ is integrable.

We prove (ii) first. So it suffices to show $h$ is integrable. Now locally we have $h(z)=(r-1) / z+A(z)$ with $A$ analytic and $r \geqq 1$. For $h(z)$ to be integrable we must find a meromorphic solution to the linear differential equation

$$
w^{\prime \prime} / w^{\prime}=(r-1) / z+A(z) \text {. }
$$

So let $v=w^{\prime}$ and rewrite this as $v^{\prime} / v=(r-1) / z+A(z)$. Then $v(z)=K z^{r-1} e^{A(z)}$; but since $r \geqq 1$ and $A$ is analytic then $v$ is holomorphic and therefore so is $w$, since $w^{\prime}=v$.

We now are left to show that an affine connection of type $\mathfrak{D}$ exists.

So let $\tau=\left\{\tau_{\alpha}\right\}$ be a holomorphic differential on $M$. Let $\chi_{\tau}=\sum_{p \in M} \nu_{p}\left(\sigma_{j}\right) \cdot p$ and let $k_{\alpha}\left(z_{\alpha}\right)=\left(d / d z_{\alpha}\right) \log \tau_{\alpha}\left(z_{\alpha}\right)$ in $U_{\alpha}$. Then by the proof of Lemma 2 we see that $k=\left\{k_{\alpha}\right\}$ is an affine connection of class $\chi_{\tau}$, and $\rho\left(\chi_{\tau}\right)=2 g-2$.

Now consider the divisor $\mathfrak{D}-\chi_{\tau}$. Since

$$
\rho\left(\mathfrak{D}-\chi_{\tau}\right)=\rho(\mathfrak{D})-\rho\left(\chi_{\tau}\right)=0,
$$

by [14] and [5] there exists an abelian differential of the third kind $\theta$ with only simple poles such that

$$
\sum_{p \in M} \operatorname{Res}(\theta, p) \cdot p=\mathfrak{D}-\chi_{\tau} .
$$

Therefore $k+\theta$ will in fact be an affine connection of class $\mathfrak{D}$.

We note that the first and third parts of our proof will be true not only for a positive divisor $\mathfrak{D}$ but for any divisor of the second kind $\chi$ with $\rho(\chi)=2 g-2$. We then have 
Corollary 5.1. Let $\chi$ be a divisor of the second kind with $\rho(\chi)=2 g-2$. Then the affine connection of class $\chi$ form a g-dimensional complex linear manifold and the integrable connections of class $\chi$ comprise a complex affine subvariety of this manifold.

Proof. The existence of the $g$-dimensional complex linear manifold of affine connections of class $\chi$ follows directly from the proof of the theorem. It thus suffices to examine the nature of the solutions to the differential equation $w^{\prime \prime} / w^{\prime}$ $=(r-1) / z+A(z)$ in the case where $r \leqq-1$. Again letting $v=w^{\prime}$ we have

$$
v(z)=K z^{r-1} e^{A(z)}=K e^{a_{0}} \sum_{n=0}^{\infty} C(n) z^{n+r-1}
$$

where $A(z)=\sum_{i=0}^{\infty} a_{i} z^{i}, C_{0}=1$, and for $n \geqq 1$

$$
C(n)=\sum_{\left(i_{1}, \ldots, i_{n}\right)} \alpha_{\left(i_{1}, \ldots, i_{n}\right)} a_{1}^{i_{1}} \cdots a_{n}^{i_{n}}
$$

and $\left(i_{1}, \ldots, i_{n}\right)$ are such that $\sum_{k=1}^{k=n} k i_{k}=n$ and $\alpha_{(1,0,0, \ldots, 0)}=1 / n !$. Thus we have

$$
w(z)=K e^{a_{0}} \sum_{n=0}^{\infty} C(n) \int z^{n+r-1} d z .
$$

This is meromorphic if and only if $C_{-r}=C_{|r|}=0$. Therefore, in general, for each affine connection $h$, there exists a polynomial

$$
H_{|r|} \in C\left[x_{1}, \ldots, x_{r}\right]
$$

such that $h$ is integrable iff $H_{|r|}\left(a_{1}, \ldots, a_{r}\right)=0$ where $a_{1}, \ldots, a_{r}$ are defined as above.

So let $h=\left\{h_{\alpha}\right\}$ be an affine connection of class

$$
\chi=\sum_{j=1}^{n}\left(r_{j}-1\right) p_{j}+\sum_{j=1}^{\sigma}\left(\nu_{j}-1\right) \cdot s_{j}
$$

and let ${ }_{1} \phi, \ldots,{ }_{g} \phi$ be a basis for the holomorphic differentials on $M$. Now for each $\boldsymbol{t} \in \boldsymbol{C}^{g}$, let ${ }_{t} k=h+\boldsymbol{\phi} \cdot \boldsymbol{t}$, where $\boldsymbol{\phi}=\left({ }_{1} \phi, \ldots,{ }_{g} \phi\right)$. We then see immediately that ${ }_{t} k$ is an affine connection of class $\chi$ and that therefore $I F_{1}(M, \chi)=$ integrable connections of class $\chi\}$ is equivalent to $\left\{\boldsymbol{t} \in \boldsymbol{C}^{g} \mid{ }_{t} k\right.$ is integrable around $s_{j}$ for $\left.j=1, \ldots, \sigma\right\}$. Now for any $\boldsymbol{t} \in \boldsymbol{C}^{g}$ we note that around $s_{j}$ with $z_{\alpha}\left(s_{j}\right)=0$ we have

$$
{ }_{t} k\left(z_{\alpha}\right)=\frac{\nu_{j}-1}{z_{\alpha}}+A\left(z_{\alpha}\right)+\boldsymbol{\phi} \cdot \boldsymbol{t}=\frac{\nu_{j}-1}{z_{\alpha}}+\sum{ }_{t} k_{m}(\alpha) z_{\alpha}^{m},
$$

and, in general,

$$
{ }_{i} k_{m}(\alpha)=A_{m}(\alpha)+\phi_{m}(\alpha) \cdot t
$$

where $A_{m}(\alpha), \phi_{m}(\alpha)=\left({ }_{1} \phi_{m}(\alpha), \ldots,{ }_{g} \phi_{m}(\alpha)\right)$ are simply the coefficients of $z_{\alpha}^{m}$ in the Taylor expansion around $z_{\alpha}\left(s_{j}\right)=0$ of $A(\alpha)$ and $\phi(\alpha)$ respectively. Thus there exists a polynomial $L_{j} \in C\left[x_{1}, \ldots, x_{g}\right]$ such that $H_{\left|v_{j}\right|}\left({ }_{t} k_{1}, \ldots,{ }_{t} k_{\left|v_{j}\right|}\right)=0$ iff $L_{j}(t)=0$. So ${ }_{t} k$ is integrable around $s_{j}$ iff $L_{j}(t)=0$; and therefore if we now let

$$
{ }_{a} V^{x}=\left\{\boldsymbol{t} \in \boldsymbol{C}^{g} \mid L_{j}(\boldsymbol{t})=0 \text { for } j=1, \ldots, \sigma\right\},
$$

then $I F_{1}(M, \chi) \approx{ }_{a} V^{\chi}$, which is the required subvariety. 
COROLlaRY 5.2. Suppose $\chi=\sum_{j=1}^{n}\left(r_{j}-1\right) \cdot p_{j}+(\nu-1) \cdot s$. Then,

(i) if $s$ is not a hyperelliptic Weierstrass point or $v$ is even, ${ }_{a} V^{x} \neq \varnothing$ and is in fact a union of hypersurfaces in $\boldsymbol{C}^{g}$; and

(ii) if $\nu$ is odd and $s$ is a hyperelliptic Weierstrass point, then if ${ }_{a} V^{x} \neq \varnothing$ it is either $C^{g}$ or a union of hypersurfaces in $C^{g}$.

Proof. From the proof of Corollary 5.1 we see that ${ }_{a} V^{x}=\left\{t \in C^{g} \mid L(t)=0\right\}$ where in our case $L(t)=H_{|v|}\left({ }_{t} k_{1}, \ldots,{ }_{t} k_{|v|}\right)$. Now if $s$ is not a hyperelliptic Weierstrass point, the basis ${ }_{1} \phi, \ldots,{ }_{g} \phi$ of abelian differentials used in constructing ${ }_{t} k$ could have been chosen so that ${ }_{2} \phi$ was the only term with nonzero coefficient of $z_{\alpha}^{\prime}$. Thus ${ }_{t} k_{1}=a_{1}+{ }_{2} \phi_{1} \cdot t_{2}$ and we can assume ${ }_{2} \phi_{1}=1$. Thus our polynomial $L(t)$ has leading term $(1 /|\nu| !) t_{2}^{1}+$ other lower order terms and this clearly shows that ${ }_{a} V^{x}$ is a union of hypersurfaces in $\boldsymbol{C}^{g}$.

Furthermore even if $s$ is a hyperelliptic point then it is still impossible for any basis of the abelian differentials to have all coefficients of both $z_{\alpha}^{1}$ and $z_{\alpha}^{2}$ zero. If some abelian differential has a nonzero $z_{\alpha}^{1}$ coefficient the above construction shows that ${ }_{a} V^{x}$ is a union of hypersurfaces. However even if all the $z_{\alpha}^{1}$ coefficients are zero at least one $z_{\alpha}^{2}$ coefficient is nonzero. We can thus always rechoose the basis so that ${ }_{2} \phi$ is the only differential in the basis with ${ }_{2} \phi_{2} \neq 0$, and we can assume ${ }_{2} \phi_{2}=1$. Then if $\nu_{1}=-2 m$ for some $m \geqq 1, L(t)$ would have leading term $(1 / m !) t_{2}^{m}$ and again ${ }_{a} V^{x}$ would be a union of hypersurfaces in $C^{g}$. However if $\nu_{1}$ is odd it is possible that $L(t)$ reduces to a constant so that if $L(t)=k$ then if $k=0,{ }_{a} V^{x}=C^{g}$, if $k \neq 0$ then ${ }_{a} V^{x}=\varnothing$ and otherwise we again have a union of hypersurfaces.

What this corollary shows us is that on any Riemann surface if we avoid a "suitably small" set of points we will always have ${ }_{a} V^{x} \neq \varnothing$.

Using techniques of [10] and [11] this can be generalized to

Corollary 2.6.4. Suppose

and

$$
\chi=\sum_{j=1}^{j=n}\left(r_{j}-1\right) \cdot p_{j}+\sum_{j=1}^{j=\sigma}\left(\nu_{j}-1\right) \cdot s_{j}
$$

$$
0<\left|\sum_{j=1}^{j=\sigma}\left(v_{j}-1\right)\right| \leqq g-1 .
$$

Then there exists an analytic subset $A \subset M^{\sigma(x)}$ of codimension $\geqq 1$ such that if $\left(s_{1}, \ldots, s_{\sigma}\right) \in M^{\sigma}-A$ then ${ }_{a} V^{x} \neq \varnothing$ and ${ }_{a}^{0} V^{x}$ has dimension $g-\sigma(\chi)$, where ${ }_{a}^{0} V^{x}$ is the submanifold of regular points of ${ }_{a} V^{x}$.

However we will pursue the subject no further.

6. Examples. To indicate the wealth of new structures now apparent on Riemann surfaces we briefly examine the case of surfaces of genus 2 . Let $M$ be such a surface and suppose $\mathfrak{D}=1 \cdot p_{1}+1 \cdot p_{2}$ is a divisor on $M$ with $p_{1}$ possibly equal to $p_{2}$. Then $\rho(\mathcal{D})=2=2 g-2$ and the space of regular affine structures with branching order $\mathscr{D}$ is just $C^{2}$. Furthermore the space of distinct positive divisors on $M$ of total branching order 2 is just $S M^{2}$, the 2 -fold symmetric product of $M$ (that is, 
$M^{2} / \sim$ where $\left(a_{1}, a_{2}\right) \sim\left(b_{1}, b_{2}\right)$ iff $\left\{a_{1}, a_{2}\right\}=\left\{b_{1}, b_{2}\right\}$ as sets). Thus any Riemann surface of genus 2 has associated to it the space $S M^{2} \times C^{2}$ of distinct regular branched affine structures.

If we now examine the projective structures associated to $M$ we find they form a subvariety ${ }_{p} V_{\mathfrak{D}}$ of $C^{5}$ containing a 3-dimensional submanifold ${ }_{p} V_{\mathfrak{D}}^{0}$. Thus the space of distinct branched projective structures of total branching order 2 form the fiber space ${ }_{p} V_{2}=\left\{\left(\mathfrak{D}, V_{\mathfrak{D}}\right) \mid \mathfrak{D} \in S M^{2}\right\}$ and this space will itself contain a submanifold of dimension 5 , namely ${ }_{p} V_{2}^{0}=\left\{\left(\mathfrak{D},{ }_{p} V_{\mathfrak{D}}^{0}\right) \mid \mathfrak{D} \in S M^{2}\right\}$. Thus it is immediately apparent that whereas in the case of $g=1$ all projective structures of order $2 g-2=0$ reduce to affine structures $[1, \S 9.3]$, this is decidedly not the case when $g=2$. In a forthcoming article we hope to apply some of this additional structure to study the holomorphic vector bundles of rank 2 on a Riemann surface [2].

\section{BIBLIOGRAPHY}

1. R. C. Gunning, Lectures on Riemann surfaces, Princeton Math. Notes, Princeton Univ. Press, Princeton, N. J., 1966. MR 34 \#7789.

2. - Lectures on vector bundles over Riemann surfaces, Univ. of Tokyo Press, Tokyo; Princeton Univ. Press, Princeton, N. J., 1967. MR 37 \#5888.

3. - Special coordinate coverings of Riemann surfaces, Math. Ann. 170 (1967), 67-86. MR 34 \#7790.

4. - Connections for a class of pseudogroup structures, Proc. Conference Complex Analysis (Minneapolis, 1964), Springer, Berlin and New York, 1965, pp. 186-194. MR 31 \#2684.

5. L. Bers, Simultaneous uniformization, Bull. Amer. Math. Soc. 66 (1960), 94-97. MR 22 \#2694.

6. - Uniformization by Beltrami equations, Comm. Pure Appl. Math. 14 (1961), 215-228. MR 24 \#A2022.

7. I. Kra, Deformations of Fuchsian groups, Duke Math. J. (to appear).

8. - On affine and projective structures on Riemann surfaces, J. Analyse Math. 22 (1969), 285-298.

9. M. Schiffer and N. S. Hawley, Connections and conformal mapping, Acta Math. 107 (1962), 175-274. MR 32 \#202.

10. H. Röhrl, On holomorphic families of fibre bundles over the Riemannian sphere, Mem. Coll. Sci. Univ. Kyoto Ser. A Math. 33 (1960/61), 435-477. MR 24 \#A1728.

11. Th. Meis, Die minimale Blätterzahl der Konkretisierung einer kompakten Riemannschen Fläche, Schr. Math. Inst. Univ. Münster No. 16 (1960). MR 26 \#5158.

12. W. E. Boyce and R. C. Di Prima, Elementary differential equations and boundary value problems, Wiley, New York, 1965. MR 31 \#3651.

13. E. Hille, Lectures on ordinary differential equations, Addison-Wesley, Reading, Mass., 1969. MR 40 \#2939.

14. G. Springer, Introduction to Riemann surfaces, Addison-Wesley, Reading, Mass., 1957. MR 19. 1169.

Department of Mathematics, Princeton University, Princeton, New Jersey 08540

Department of Mathematics, Newark College of Engineering, Newark, New Jersey 07102

Current address: Department of Mathematics, University of Massachusetts, Amherst, Massachusetts 01002 